\title{
Aircraft Wake-Vortex Evolution in Ground Proximity: Analysis and Parameterization
}

\author{
Frank Holzäpfel $*$ and Meiko Steen $\ddagger$ \\ DLR, German Aerospace Research Center, Oberpfaffenhofen, 82234 Weßling, Germany
}

DOI: $\underline{10.2514 / 1.23917}$

\begin{abstract}
Field measurement data of 288 wake-vortex pairs and respective environmental conditions acquired at Frankfurt Airport by means of light detection and ranging, sound detection and ranging/radio acoustic sounding system, and ultrasonic anemometer are used to analyze wake-vortex behavior in ground proximity. Exceptional cases with strong rebounds caused by detached shear layers and obstacles are introduced and estimates of the time needed to clear the runway from wake vortices by advection are provided. The impact of turbulence and crosswind on wakevortex decay proves to be weak, whereas already light crosswind turns out to be sufficient to cause pronounced asymmetric rebound characteristics. Based on the analyses vortex decay and rebound characteristics are parameterized and implemented into the probabilistic two-phase aircraft wake-vortex model. Deterministic and probabilistic prediction skill of the enhanced vortex model are assessed. Comparison to wake predictions out of ground effect indicates that in ground effect 1) the rapid-decay phase progresses slower, 2) wake-vortex evolution can be predicted with improved accuracy, and 3) fair prediction skill requires only limited environmental data.
\end{abstract}

\section{Nomenclature}

$\begin{array}{ll}A & = \\ b & = \\ C & = \\ q & = \\ R & = \\ T & = \\ t & = \\ u & = \\ v & = \\ w & = \\ y & = \\ z & = \\ \Gamma & = \\ \epsilon & = \\ \sigma & = \\ v & =\end{array}$

Subscript

\begin{tabular}{|c|c|c|}
\hline$g$ & $=$ & minimum height above ground \\
\hline$l$ & $=$ & lower limit \\
\hline lat & $=$ & lateral \\
\hline meas & $=$ & measured \\
\hline prim & $=$ & primary \\
\hline sec & $=$ & secondary \\
\hline$u$ & $=$ & upper limit \\
\hline vert & $=$ & vertical \\
\hline 0 & $=$ & initial value \\
\hline 00 & $=$ & value at minimum height \\
\hline 1 & $=$ & first decay phase \\
\hline 2 & $=$ & second decay phase \\
\hline $5-15$ & $=$ & 5 to $15 \mathrm{~m}$ average \\
\hline
\end{tabular}

Presented as Paper 1077 at the 44th AIAA Aerospace Sciences Meeting and Exhibit, Atmospheric and Space Environments, Reno, NV, 9-12 January 2006; received 15 March 2006; revision received 28 June 2006; accepted for publication 29 June 2006. Copyright (C) 2006 by the American Institute of Aeronautics and Astronautics, Inc. All rights reserved. Copies of this paper may be made for personal or internal use, on condition that the copier pay the $\$ 10.00$ per-copy fee to the Copyright Clearance Center, Inc., 222 Rosewood Drive, Danvers, MA 01923; include the code $\$ 10.00$ in correspondence with the CCC.

*Research Scientist, Institut für Physik der Atmosphäre; frank.holzaepfel@dlr.de.

${ }^{\dagger}$ Student, Institut für Physik der Atmosphäre.

\section{Superscript}

$* \quad=$ normalized by initial vortex parameters $b_{0}, t_{0}, w_{0}, \Gamma_{0}$

$\quad$ normalized by probabilistic bounds

\section{Introduction}

$\mathbf{P}$ ERSISTENT aircraft trailing vortices entail aircraft separation distances that degrade aviation efficiency at busy airports. Because of comprehensive research efforts on both sides of the Atlantic, wake-vortex advisory systems that aim to safely adjust aircraft separations based on wake-vortex behavior prediction and monitoring seem to come into reach [1].

The largest probability to encounter wake vortices shed by preceding aircraft prevails during final approach in ground proximity $[2,3]$. There clearance of the flight corridor by descent and advection is significantly restricted: stalling or rebounding vortices may not clear the flight corridor vertically and weak crosswinds may be compensated by vortex-induced lateral transport which may prevent the vortices to leave the corridor laterally. Moreover, the possibilities of the pilot to counteract the imposed rolling moment are restricted due to the low height of the aircraft above ground. Therefore, reliable wake-vortex prediction in ground proximity constitutes a vital requirement within a wake-vortex advisory system.

The interaction of wake vortices with the ground was first considered in [4]. At first, the vortices induce a boundary layer (vorticity layer) at the solid surface which causes the wake vortices to diverge driven by mutual velocity induction. Another descriptive explanation of the phenomenon results from arguments of mass conservation: the diverging vortices circulate ambient air from their front sides (outboard) to their back sides (inboard) which in turn displaces the vortices laterally towards the outboard side. The effect is usually modeled by introducing image vortices that replace the former partner vortex aloft. Because of an adverse pressure gradient the boundary layer may separate subsequently which leads to the formation of secondary vortices. The interaction of primary and secondary vortices causes the former to detach from the hyperbolic trajectory of classical inviscid theory and the newly formed unequal vortex pairs rebound.

This behavior was confirmed by numerical simulations [5-16], laboratory experiments $[\underline{7}, 17]$, and field measurement data $[\overline{18}, \overline{19}]$. A survey on vortex interactions with walls is given in [20]. Twodimensional simulations $[\underline{6}, \underline{8}-\underline{10}]$ indicate that crosswind shear may attenuate the formation of the secondary vortex on the luff (upwind) side whereas the secondary vortex on the lee (downwind) side is strengthened, a scenario which causes asymmetric rebound 
characteristics. Since wake-vortex decay mechanisms are in general directly related to the interaction between counter-rotating vortex pairs [21] it is fair to assume that the unequally strong secondary vortices should also provoke asymmetric decay rates in crosswind situations. That is the interaction of the lee vortex with its stronger secondary vortex should reduce the lee vortex lifetime compared with the luff vortex. Three-dimensional simulations illustrate that secondary vortices are susceptible to spatial crosswind variations [11] or short-wavelength instabilities [12] which both deform the secondary vortices and subsequently lead to reconnection of primary and secondary vortices. Linking of vortices with the ground is reported at moderate to high ambient turbulence levels [13]. All these findings suggest that considerable variability of vortex evolution has to be expected which may hamper an unambiguous analysis of fullscale wake-vortex behavior in ground proximity. Further, it is described that vortices decay more rapidly near the ground and that the decay rate is largely independent from ambient turbulence $[13,19,22]$. There is no general consensus whether wake-vortex evolution in ground proximity can $[13,14]$ be normalized by the common vortex scales, namely initial circulation $\Gamma_{0}$ and vortex spacing $b_{0}$ or not [19].

The current manuscript analyzes light detection and ranging (lidar) measurement data of 288 wake-vortex pairs collected in ground proximity at Frankfurt Airport in order to scrutinize the current understanding of ground-effect (GE) physics and to improve the parameterization of the probabilistic two-phase aircraft wakevortex model (P2P) [23-25]. Data of that measurement campaign were also used in another study [26] to investigate the predictive skill of the P2P model employing environmental parameters provided by short-term weather forecasts. In the current manuscript, first an overview on the used database is given, and then the P2P model is briefly introduced. Section IV investigates wake-vortex circulation decay statistics and the potential impact of crosswind shear and turbulence. In Sec. V vortex trajectories are analyzed with a focus on effects of crosswind, shear layers, and obstacles on rebound characteristics. Crosswind thresholds are derived which ensure that a safety corridor around the glide path is cleared from wake vortices. Section VI describes the consequential adaptations of the probabilistic components of P2P. Finally, in Sec. VII the achieved wake prediction skill in ground proximity is assessed.

\section{Wake-Vortex Database}

The wake-vortex measurement campaign WakeFRA has been accomplished at Frankfurt Airport during the period from August to December 2004. The current study focuses on lidar measurement data of 288 wake-vortex pairs collected in ground proximity during nine days in August and September. The main body of the study employs wake vortices generated by A340-300, A340-600, and B747-400 aircraft on approach to runway $25 \mathrm{~L}$ at a nominal height of $55 \mathrm{~m}$ (110 cases) and 127 approaches to runway $25 \mathrm{R}$ at a nominal height of $61 \mathrm{~m}$ (see Fig. 1). To investigate scaling effects on wakevortex evolution in ground proximity, additionally, two hours of regular traffic mix on 28 September comprising 25 different aircraft types are analyzed. Within these two hours a total of 73 aircraft landed from which 51 approaches were independent from the counts of heavy aircraft listed above.

Figure 1 shows the WakeFRA site with the locations of the employed sensors. A Meteorologische Messtechnik $\mathrm{GmbH}$ (METEK) sound detection and ranging (SODAR) with a radio acoustic sounding system (RASS) extension provided $10 \mathrm{~min}$ averaged vertical profiles of the three wind components, vertical fluctuation velocity, and virtual temperature with a vertical resolution of $20 \mathrm{~m}$. The SODAR/RASS system was complemented by an ultrasonic anemometer (USA) mounted on a $10 \mathrm{~m}$ mast. A spectral analysis of the longitudinal velocity measured by the sonic is used to estimate turbulence eddy dissipation rate (EDR) by fitting the $-\frac{5}{3}$ slope in the inertial subrange of the velocity frequency spectrum.

The 2- $\mu \mathrm{m}$ pulsed lidar system [27] scanned the measurement plane in an angle of 123 deg to the direction of the approaching aircraft (see Fig. 1, dashed line) employing elevation sectors from 0

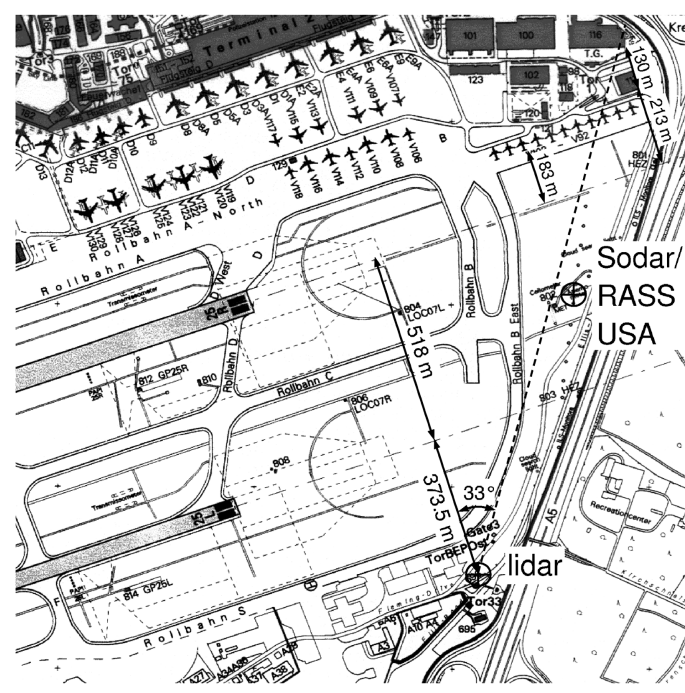

Fig. 1 WakeFRA site. Map reprinted by courtesy of Fraport AG.

to $15 \mathrm{deg}$. Along the measurement plane the ground mainly consists of grassland interrupted by a taxiway and an aircraft parking site. For the evaluation of wake-vortex properties, an interactive four-stage data processing algorithm was applied, which is described in detail in [27]. From the estimated profiles of vortex tangential velocities vortex positions and circulations were derived. Circulation data were corrected employing the cosine of the $33 \mathrm{deg}$ deviation from an observation angle perpendicular to flight direction. This correction leads to an overestimation of circulation of deformed vortices that tend to be aligned perpendicular to the viewing angle of the lidar and an underestimation for vortex segments that turn away from that viewing angle. While this effect increases the scatter of the circulation data there is no net contribution to average circulation values because overestimation and underestimation on average compensate each other exactly. Unfortunately, analysis of wakevortex properties on the $25 \mathrm{~L}$ side was hampered by nonuniform signal to noise ratios at distances of less than $500 \mathrm{~m}$ to the lidar. Therefore, the investigation of vortex behavior in-GE focuses on data from runway $25 \mathrm{R}$ whereas $25 \mathrm{~L}$ data are mainly used for validation purposes.

During the evaluation of circulation secondary vortices could be identified in about $50 \%$ of the cases. These GE vortices become manifested in peaks within the lidar velocity spectra typically situated below the primary vortices. To restrain modifications of the evaluated tangential velocities by these secondary vortices and the related increase of the scatter of the circulation data the slope of the undisturbed side of the velocity spectra is fitted to the disturbed part.

From lidar measurements also vertical profiles of eddy dissipation rate were derived from Doppler spectrum width [28]. EDR data were determined with a vertical resolution of $20 \mathrm{~m}$ and an averaging time of about $5 \mathrm{~min}$. To minimize the impact of wake turbulence, areas contaminated by wake vortices were identified and cut out prior to the estimation of EDR. A comparison of EDR data derived from lidar data to EDR data based on ultrasonic anemometer measurements indicates that the relative rms error amounts to $46 \%$ provided that the integral length scale of turbulence is larger than $90 \mathrm{~m}$. For shorter turbulence length scales lidar EDR data is generally overestimated.

\section{Wake-Vortex Model}

The methods employed for the analysis of wake-vortex behavior in ground effect and the further development of the GE parameterization of the Probabilistic Two-Phase aircraft wakevortex model $(\mathrm{P} 2 \mathrm{P})$ are directly combined. Here only the properties of $\mathrm{P} 2 \mathrm{P}$ are recapitulated which are relevant in the current context. Detailed descriptions, applications, and assessments of P2P out-ofGE are available in [23-25].

The model is formulated in normalized form where the characteristic scales are based on initial vortex separation and 

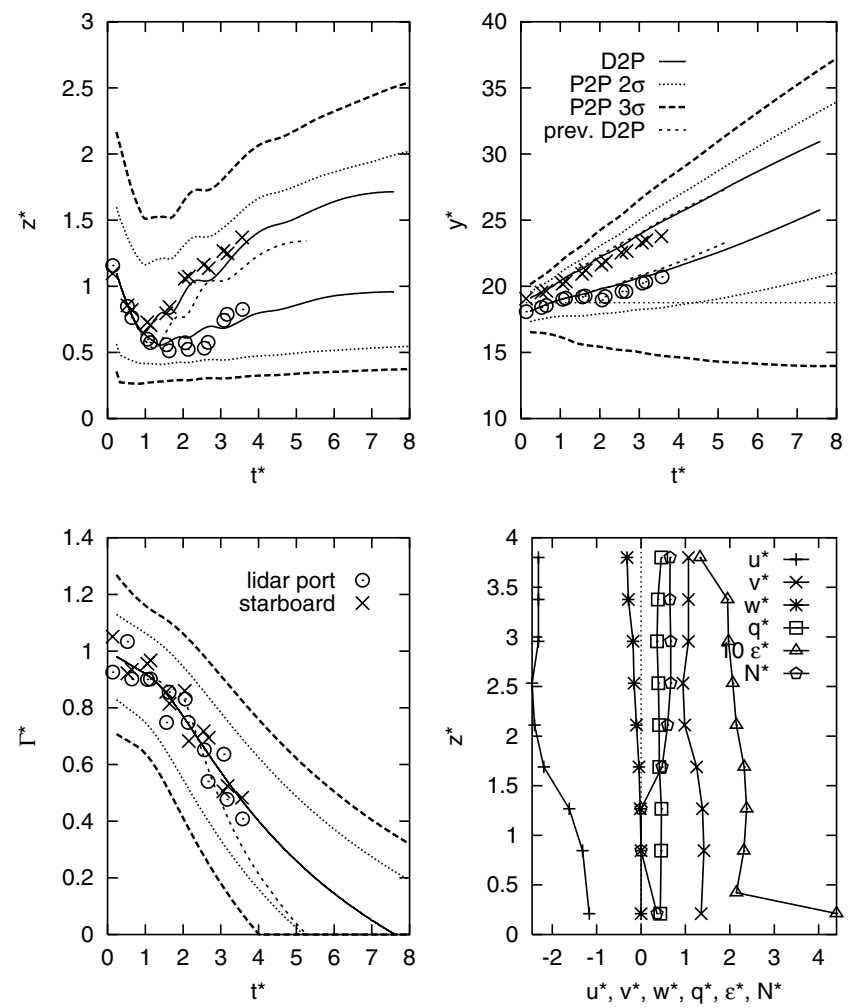

Fig. 2 Measured (symbols) and predicted (lines) evolution of normalized vertical and lateral positions and circulation.

circulation leading to the time scale $t_{0}=2 \pi b_{0}^{2} / \Gamma_{0}$. EDR is normalized according to $\epsilon^{*}=\left(\epsilon b_{0}\right)^{1 / 3} / w_{0}$, where $w_{0}=\Gamma_{0} / 2 \pi b_{0}$ denotes the initial descent speed. P2P employs a circulation, $\Gamma_{5-15}^{*}$, that is averaged over circles with radii from 5 to $15 \mathrm{~m}$.

For the prediction of circulation, the concept of two-phase circulation decay is pursued (see Fig. 2). The devised formulation results from an adaptation of the equation that describes circulation evolution of the decaying potential vortex [29] to large eddy simulation results of wake-vortex evolution in turbulent and stably stratified environments. The turbulent diffusion phase described by part 1 of Eq. (1) is followed by a rapid-decay phase represented by the full equation

$$
\Gamma_{5-15}^{*}\left(t^{*}\right)=\underbrace{A-\exp \frac{-R^{2}}{v_{1}^{*}\left(t^{*}-T_{1}^{*}\right)}}_{1}-\exp \frac{-R^{2}}{v_{2}^{*}\left(t^{*}-T_{2}^{*}\right)}
$$

Out of GE the onset time of rapid decay at $T_{2}^{*}$ depends on ambient turbulence and stratification and the respective decay rate is adjusted by the effective viscosity $v_{2}^{*}$. The constant parameters $T_{1}^{*}$ and $v_{1}^{*}$ control decay in the diffusion phase, $R$ corresponds to a mean radius, and $A$ is a constant to adjust $\Gamma_{5-15}^{*}\left(t^{*}=0\right)$. In the following, the dependence of decay parameters $T_{2}^{*}$ and $v_{2}^{*}$ on ground proximity, crosswind, and EDR is investigated and parameterized accordingly.

To consider spatiotemporal variations of vortex position and strength, which are caused primarily by turbulent transport and deformation processes, the probabilistic wake-vortex model predicts wake-vortex behavior within defined confidence intervals (see Fig. 2, solid lines denote deterministic behavior, dotted and dashed lines envelopes for probabilities of 95.4 and $99.7 \%$, respectively; deterministic predictions with previous GE model plotted with dotted lines for comparison; right below, vertical profiles of normalized environmental data). For this purpose, decay parameters $T_{2}^{*}$ and $v_{2}^{*}$ are varied in consecutive model runs and various static and dynamic uncertainty allowances are added which consider the increased scatter in turbulent environments and modified trajectories caused by tilting and rebound in wind shear situations. The obtained probabilistic envelopes can be adjusted to represent selected degrees of probability. The respective envelopes are estimated based on a training procedure [25] that relates the predicted envelopes to field measurement data. A deterministic model version termed D2P provides mean wake-vortex evolutions employing intermediate decay parameters.

\section{Circulation Evolution}

Figure 3 shows a scatter plot of the circulation evolution of a subset of 127 approaches to runway $25 \mathrm{R}$ and the respective evolution of the median value determined within a running window of width $\Delta t^{*}=1$. Circulation is normalized by the first reasonable circulation measurement. In cases where the first circulation value was obviously strongly overestimated because in the near field behind the aircraft the roll-up to a single vortex pair was not yet finalized [30], the second measurement is used as initial value. The fit of Eq. (1) to lidar measurements (in legend termed D2P) yields $T_{2}^{*}=1.23$ and $v_{2}^{*}=0.0028$

For $t^{*}>2.5$ the model fit and the median of measurements start to deviate increasingly. Figure 4 is used to illustrate by means of inverse modeling that the characteristics assumed by the fit are plausible in spite of the pronounced deviation from the median of measurements. The scatter plot in Fig. 4 is achieved by generating Gaussian random numbers around the $\overline{\mathrm{D}} 2 \mathrm{P}$ fit where in both, measurement and simulation the standard deviations of circulation values roughly amount to 0.1. Additionally, a filter (see Fig. 4, right) is applied to the synthetic scatter plot which assumes that above $\Gamma^{*}=0.7$ circulation from all vortex scans could be evaluated, whereas below $\Gamma^{*}=0.3$ no evaluation would be possible anymore and a linear transition in between. Both, the scatter plots of measurement data and data

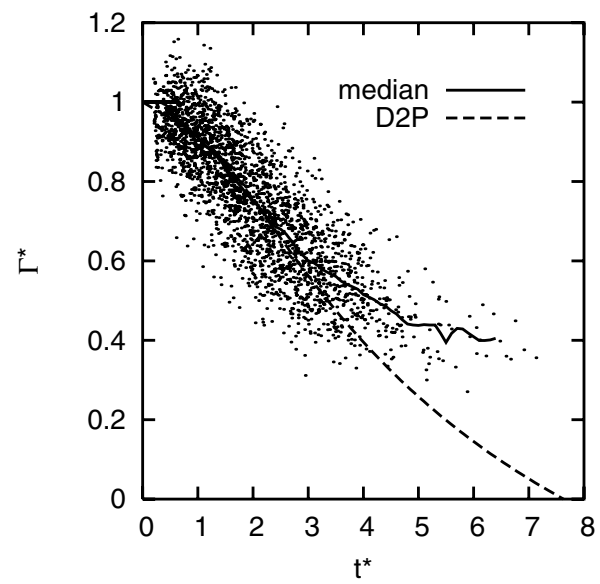

Fig. 3 Scatter plot of normalized lidar circulation data with median and fit of Eq. 1 .

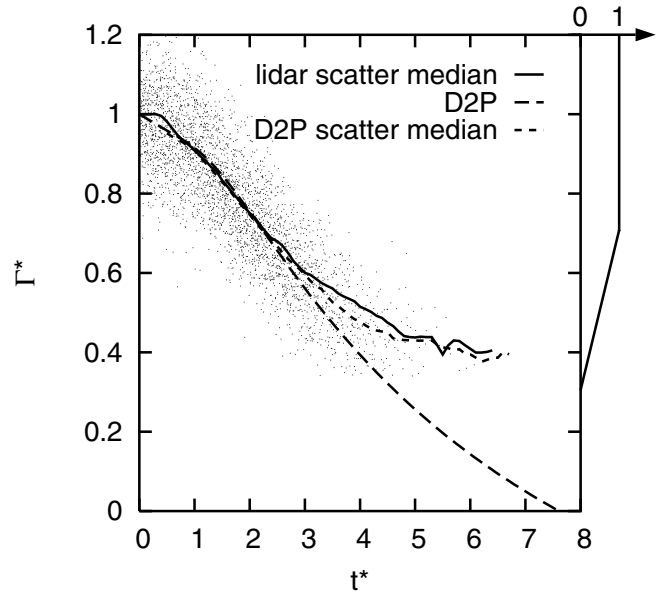

Fig. 4 Synthetic circulation scatter plot derived from D2P decay model. Applied filter sketched right. 


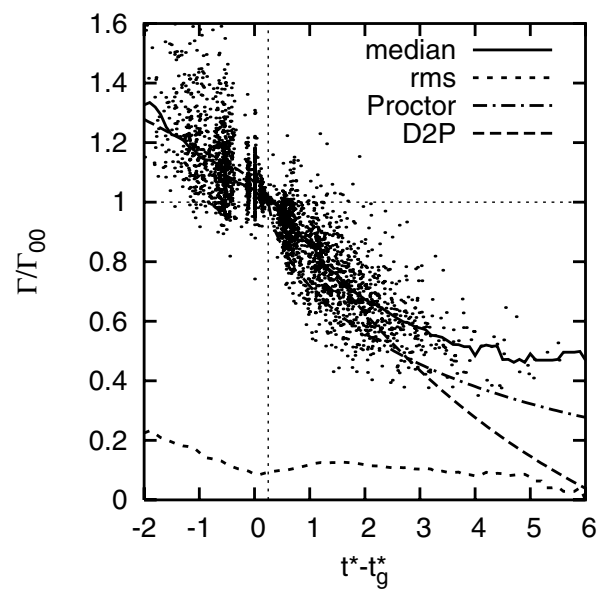

Fig. 5 Circulation evolution normalized as suggested by Proctor et al. [13].

achieved by inverse modeling and the resulting medians have very similar characteristics. This illustration indicates that as soon as circulation evaluation of a few cases is not possible any more (due to progressive erosion of the typical wake-vortex structure) the data median is biased towards higher circulation values. Finally, the median approaches the circulation value of the last successful circulation evaluation and the real statistics actually situated below is masked. Note that the threshold for the estimation of circulation from lidar data depends on the degree of erosion of the vortices, signal to noise ratio, circulation magnitude, and turbulence intensity of the environmental flow [30]. So the relative detection limit of circulation is lower for weaker turbulence whereas the absolute detection limit declines for smaller aircraft.

Large eddy simulations (LES) of wake vortices in ground proximity [13] indicate that appropriate normalization reduces vortex sensitivity to initial circulation, initial separation, and generating height. Figure 5 depicts circulation evolution employing the normalization derived from the parameterization of the LES results [13]. For this purpose the time coordinate is shifted by the time of minimum height above ground $t^{*}=t_{g}^{*}$ and circulation is normalized by $\Gamma_{00}=\Gamma\left(t_{g}^{*}+0.25\right)$. In contrast to the simulations reported in [13] this normalization does not reduce the scatter of circulation data measured in GE. The standard normalization (as applied in Fig. 3) yields a standard deviation of $\sigma_{\Gamma^{*}}=0.091$ whereas the GE normalization achieves $\sigma_{\Gamma^{*}}=0.11$.

The formula for circulation decay in GE suggested by [13] follows the calculated median with a certain offset. It appears that the suggested model initially tends to decay too abruptly, which potentially could be due to the fact that in the underlying LES the integration of circulation in the 5-15 m range might enclose counterrotating secondary vorticity induced at the ground. Recalling the discussion of Fig. 4 it further appears that the suggested model seems to underestimate decay at late times. This underestimation can be avoided by the constraint that the decay rate is never allowed to become less than a predetermined slope [31].

To investigate the impact of crosswind shear and turbulence on vortex decay in GE the following procedure is applied. For onset of GE decay we assume that $T_{2}^{*}=t_{g}^{*}-1$. The characteristics of Eq. (1) then lead to an effective onset of rapid decay at $t^{*}=t_{g}^{*}+0.15$ which is close to the parameterization suggested in [13] employing $t^{*}=t_{g}^{*}+0.25$. (This adjustment of $T_{2}^{*}$ applies to the analysis of measurement data. For wake-vortex predictions with the P2P model we use $T_{2}^{*}=t^{*}\left(z^{*}=1\right)$ which likewise leads to an effective onset of rapid decay at about 0.15 time units after the vortices have passed the minimum height. That is, the vortices need approximately one time unit to descend from a height of one vortex spacing to the minimum height above ground.) Then $v_{2}^{*}$ values are determined by leastsquares fits to the data of every aircraft approach. The resulting $v_{2}^{*}$ values are then plotted against potential impact parameters such as crosswind or eddy dissipation rate. For example, for crosswind $v^{*}$, the final fit is determined by calculating the median value of $v_{2}^{*}\left(v^{*}\right)$

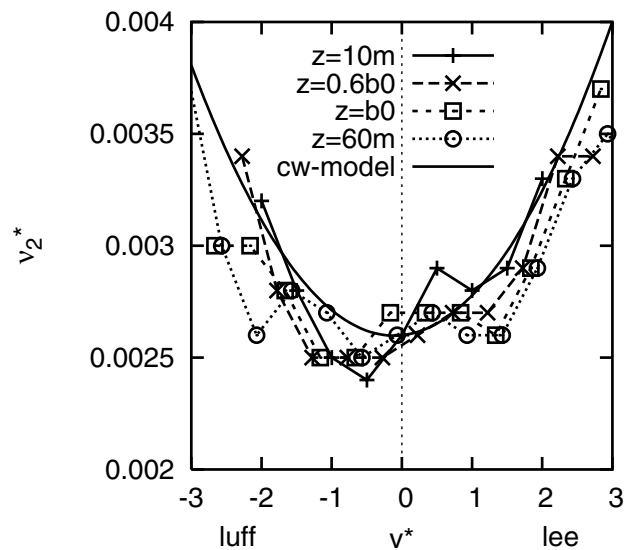

Fig. 6 Effective viscosity $v_{2}^{*}$ as function of crosswind at different heights together with respective parameterization (cw model).

within a running window of width $\Delta v^{*}=1$. The resulting fits based on crosswinds determined at different heights are shown in Fig. 6.

We assume that the physical mechanisms that modify vortex $\overline{\mathrm{GE}}$ behavior in crosswind situations are related to the interaction of crosswind shear with, primarily, the formation of secondary vortices and, secondary, the direct interaction of shear with primary vortices. Since, on one hand, crosswind shear at low altitude was not directly resolved by measurements but, on the other hand, the no-slip condition at the ground provides an implicit relation between measured crosswinds and crosswind shear, we use crosswinds measured at different heights to characterize crosswind shear. The candidate heights comprise $z=10 \mathrm{~m}$ to test the suitability of standard meteorological instrumentation, $60 \mathrm{~m}$ to represent the height of vortex generation, $b_{0}$ as the standard wake-vortex length scale, and $0.6 b_{0}$ for crosswinds at the average minimum descent height.

Figure 6 distinguishes the effects of crosswind on luff (upwind) and lee (downwind) vortex, where the crosswind directed from luff to lee vortex has a positive sign. The figure indicates that the decay rate mainly increases with crosswind magnitude and, surprisingly, is only little skewed. Crosswinds measured at different heights lead to similar results. The fit of $v_{2}^{*}$ to crosswind measured at $10 \mathrm{~m}$ height and at $z^{*}=0.6 b_{0}$ via a quadratic polynomial function yields

$$
v_{2, \text { luff(lee) }}^{*}=0.0026-(+) 3.27 \times 10^{-5} v^{*}+1.45 \times 10^{-4} v^{* 2}
$$

Figure 7 shows the normalized eddy dissipation rate as function of normalized crosswind magnitude based on anemometer measurements at $10 \mathrm{~m}$ height together with the respective linear fit. Since crosswind is well correlated with EDR we assume that the accelerated decay observed at higher crosswind magnitudes is actually rather related to increased turbulence. Figure $\underline{8}$ shows fits of

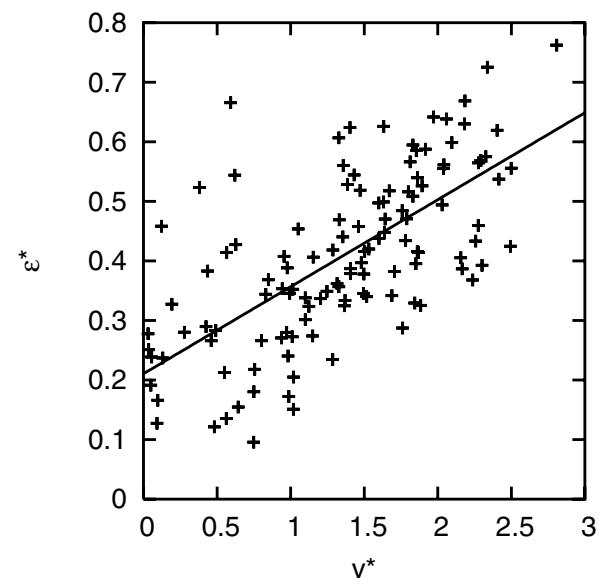

Fig. 7 Normalized eddy dissipation rate as function of normalized crosswind magnitude. 


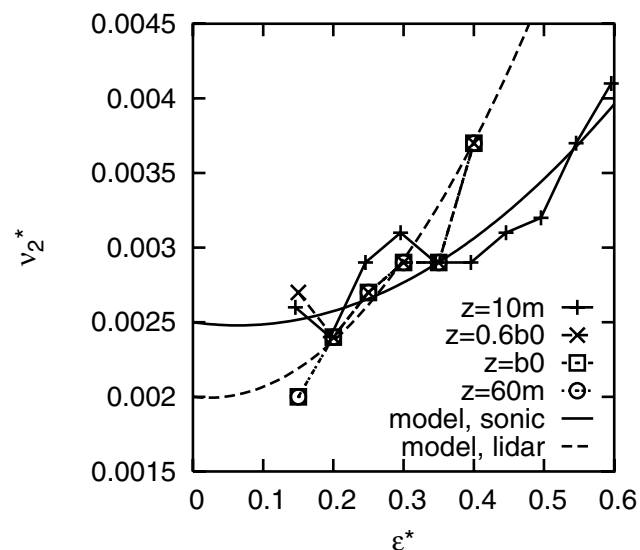

Fig. 8 Effective viscosity $v_{2}^{*}$ as function of EDR evaluated at different heights.

effective viscosity $v_{2}^{*}$ as function of normalized EDR determined by lidar at heights of $0.6 b_{0}, b_{0}$, and $60 \mathrm{~m}$ and by sonic at $10 \mathrm{~m}$ height together with the respective parameterizations. Complementary to [13], which demonstrates that vortex decay rate has minor sensitivity to ambient turbulence within an EDR-range of $0.079 \leq \epsilon^{*} \leq 0.14$, we observe a clear correlation of decay and turbulence at larger EDR values. The corresponding parameterization based on EDR evaluated from data measured by the sonic anemometer $(z=10 \mathrm{~m})$ reads

$$
v_{2}^{*}=0.0025-0.00066 \epsilon^{*}+0.00516 \epsilon^{* 2}
$$

whereas EDR based on lidar yields

$$
\nu_{2}^{*}=0.002-0.00053 \epsilon^{*}+0.012 \epsilon^{* 2}
$$

An assessment of the prediction skill achieved with Eqs. (2-4) is presented below.

\section{Trajectories}

\section{A. Rebound Characteristics}

The statistics of maximum descent and maximum rebound height as function of crosswind measured at $z^{*}=0.6 b_{0}$ (231 overflights, 5210 vortex observations) delineated in Fig. 9 indicate clear differences between luff and lee vortices. The absolute lowest descent heights and highest rebound heights as well as the respective medians clearly show lower values for the luff vortex compared with the lee vortex. This suggests that for the luff vortex the generation of the secondary (GE) vortex is delayed and its strength is reduced as it is observed in simulations $[6,8-10]$. For the lee vortex these trends are reversed. The median values indicate that, evidently, relatively weak crosswinds are sufficient to generate the observed asymmetry of vortex rebound, whereas strong crosswinds intensify the effects

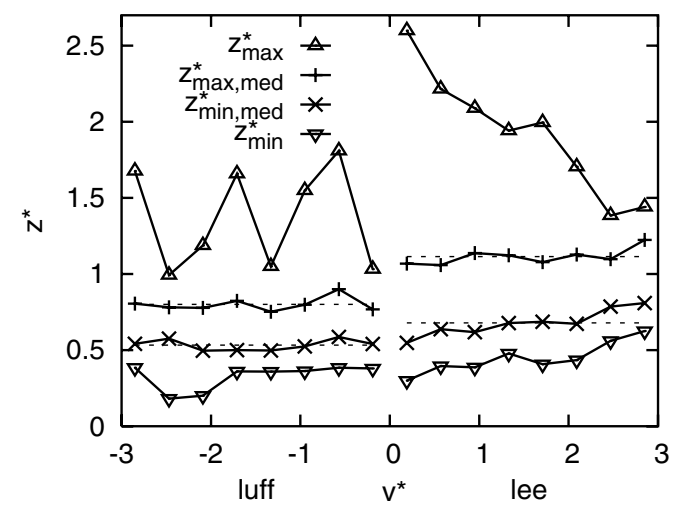

Fig. 9 Statistics of maximum descent and maximum rebound height as function of crosswind.
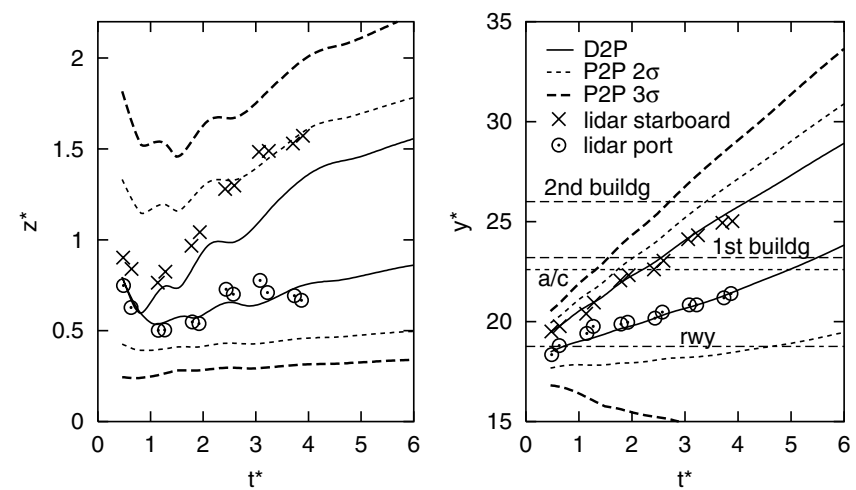

Fig. 10 Impact of obstacles on vortex rebound.

only marginally. The decrease of maximum rebound heights with increasing crosswinds for the lee vortices is presumably caused by reduced vortex lifetimes and vortex observation times at increased crosswind and turbulence levels. Analytical considerations [20] suggest that the formation of the upwind secondary vortex and the sequential luff vortex rebound is suppressed at crosswinds above four descent speeds. Because of the restricted crosswind range, we could not provide evidence of this phenomenon.

The data base contains various cases with instructive rebound characteristics from which two are sketched in the following. Figure 10 depicts a case where the lee vortex is advected with an almost constant crosswind of $v^{*}=1.5$ beyond a parking site for regional jets and a building of $9.7 \mathrm{~m}$ height (see Fig. 1). Several similar cases indicate that the vortices are deflected upwards as soon as they encounter obstacles like aircraft or buildings which here is the case after $t^{*}=2$. Maximum observed rebound altitudes amount to $2 b_{0}$. We argue that the combination of two effects causes the intensification of the rebound: obstacles may act as elevated ground and they may generate wind shear above. Similar characteristics were observed by lidar and simulated by large eddy simulation for an extreme rebound which was triggered by a group of trees at LondonHeathrow Airport [32]. Vortices that traveled beyond the parking site were excluded from the statistics presented in Fig. 9.

Two cases were identified where a shear layer situated above the vortices apparently intensifies the rebound. Maximum observed rebound height was $2.6 b_{0}$ (see Fig. 9). Figure 11 right indicates almost no crosswind below $z^{*}=1.3$, whereas above a crosswind shear rate of $\partial v^{*} / \partial z^{*}=1.8$ was diagnosed. Also the axial wind component features considerable shear in the same height range. Note that also axial shear can cause vortex rebound [25]. Possibly, as a consequence, the lee vortex noticeably exceeds the predicted 3- $\sigma$ envelope. A closer inspection of the lidar data indicates that the signature of the secondary vortex becomes weaker for $z^{*}>1$ and becomes stronger again when it approaches the shear layer. It is not clear whether the GE secondary vortex is intensified by the wind shear or if a new secondary vortex forms from shear layer vorticity. The luff vortex evolves too close to the lidar which prevents proper data analysis.
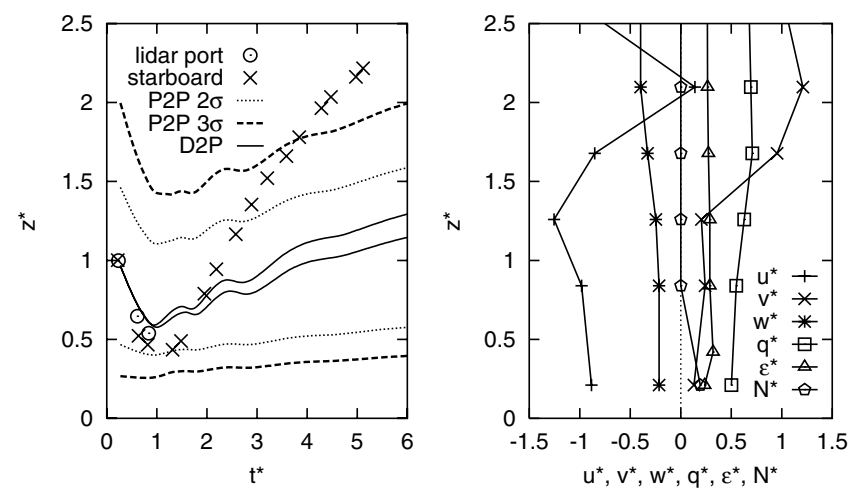

Fig. 11 Impact of an elevated shear layer on vortex rebound. 
To the authors' knowledge this particular wake-vortex/shear layer topology has as yet neither been observed in experiments nor investigated by numerical simulation. Therefore, only the unusual rebound height and the fact that shear layer and wake-vortex exhibit opposite signed vorticity, which is necessary to explain the intensified rebound, support the conclusion that a shear layer above may amplify wake-vortex rebound in ground proximity.

\section{B. Windward Stall}

A potentially most dangerous situation for following aircraft arises from wake vortices hovering above the runway because the selfinduced lateral propagation velocity of the luff vortex is just compensated by the crosswind. To identify crosswind ranges that support or exclude windward vortex stall, the relation between lateral transport of the wake vortices and crosswind is analyzed based on the 231 aircraft approaches. For this purpose, the mean lateral transport velocity of each vortex is estimated employing the first and the last measured lateral vortex position. An inspection of many individual cases suggests that the underlying assumption of constant lateral propagation speed provides a reasonable approximation for the compilation of the current transport velocity statistics.

Figure 12 displays the distribution of analyzed transport velocities as a function of crosswind at $z^{*}=0.6 b_{0}$ together with the respective quadratic polynomial fits. In the figure crosswinds directed from luff to lee vortex have positive signs. The fits indicate that the average vortex-induced propagation velocity amounts to about $0.5 \mathrm{~m} / \mathrm{s}$ for calm situations and becomes larger for stronger crosswind. Note that this average velocity comprises also the very early and late vortex evolution phases in which the vortex-induced lateral transport is zero or small, respectively. The weak though discernible observed nonlinear correlation of transport and crosswind velocities indicates that either the crosswind profiles are not self-similar at different crosswind magnitudes (that is crosswind profiles can not be characterized by measurements at a single altitude) and/or that vortex rebound depends on crosswind magnitude. Figure 12 compiled with normalized quantities (not shown) indicates that the crosswind which on average just compensates vortex-induced propagation speed amounts to $v^{*}=0.5$.

To estimate a crosswind threshold above which the runway is cleared from wake vortices the definition of the safety area of a runway used within the Frankfurt wake vortices warning system [33] is employed. This safety corridor assumes a maximum lateral deviation of the aircraft from the runway centerline of $\pm 15 \mathrm{~m}$ plus a $30 \mathrm{~m}$ distance between vortex core and aircraft fuselage. Thus, the luff vortex has to travel at most $b_{0} / 2+2 \cdot 15 \mathrm{~m}+30 \mathrm{~m} \approx 84 \mathrm{~m}$ to quit the runway. Assuming a $125 \mathrm{~s}$ time separation ( $5 \mathrm{NM}$ ) between leading and following aircraft yields a vortex transport velocity threshold of $0.67 \mathrm{~m} / \mathrm{s}$ above which the vortices have left the safety corridor even when they were generated at the upwind edge of the corridor. The rightmost symbol in the dark gray area in Fig. 12 indicates that the crosswind must exceed $2.6 \mathrm{~m} / \mathrm{s}(2.5 \mathrm{~m} / \mathrm{s})$ at $z^{*}=0.6 b_{0}(10 \mathrm{~m})$ to satisfy the criterion. For a radar separation of $2 \mathrm{NM}$ the vortex transport velocity threshold amounts to $1.7 \mathrm{~m} / \mathrm{s}$

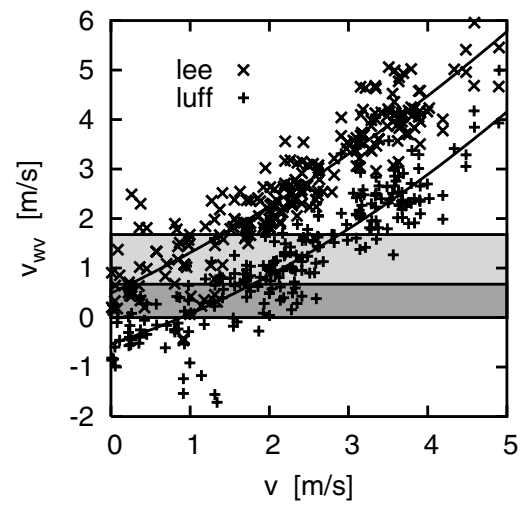

Fig. 12 Scatter plot of average lateral transport velocities of luff and lee vortices as function of crosswind. (light gray area) with respective crosswind thresholds of $3.6 \mathrm{~m} / \mathrm{s}$ $(3.3 \mathrm{~m} / \mathrm{s})$ at $z^{*}=0.6 b_{0}(10 \mathrm{~m})$.

Note that in the cases which are situated rightmost in Fig. 12 and are used to derive the crosswind limits, the crosswinds actually sensed by the vortices were smaller than the measured crosswinds which enter the analysis. That is, the found crosswind thresholds already imply uncertainties due to measurement error and spatiotemporal deviations between the winds prevailing at the sites of vortex observation and meteorological instrumentation. Further, the estimate assumes that all luff vortices are generated at the outmost windward side of the safety corridor. Actually, all observed vortices have quit the safety area at crosswind magnitudes below the found thresholds. Finally, it should be noted that the derived crosswind thresholds may differ for other vortex generation heights where safety corridor dimensions, vortex-induced propagation distances, and meteorological conditions deviate from the investigated situation.

\section{Parameterization}

The effect of the ground on vortex trajectories in P2P so far has been modeled following the approach of Robins, Delisi, and Greene [34] which is illustrated in Fig. 13. Image vortices are introduced when the primary vortices have reached a height of $1.5 b_{0}$ above ground. At a height of $z_{\mathrm{sec}}=0.6 b_{0}$ counter-rotating ground-effect vortices and their respective image vortices are introduced at an angle of $45 \mathrm{deg}$ inboard below the primary vortices at a distance of $0.4 b_{0}$. Another pair of secondary vortices with images is introduced when the first pair has rotated $180 \mathrm{deg}$ around the primary vortices. The strength of the secondary vortices is a function of the rotation angle and reaches a maximum of $\Gamma_{\mathrm{sec}}=-0.4 \Gamma_{\text {prim }}$ after being traveled $90 \mathrm{deg}$.

The parameters $z_{\mathrm{sec}}^{*}$ and $\Gamma_{\mathrm{sec}} / \Gamma_{\text {prim }}$ are readjusted based on the statistics depicted in Fig. 9, visual inspection of many cases, and on results of the scoring procedure described further below. For $z_{\mathrm{sec}}^{*}$ the original height of 0.6 is kept for the luff vortex and it is increased to 0.8 for the lee vortex (see Fig. 14). The linear transition of $z_{\mathrm{sec}}^{*}$ and $\Gamma_{\mathrm{sec}} / \Gamma_{\text {prim }}$ between $v^{*}= \pm 1$ accounts for the relatively abrupt transition between the rebound characteristics of the lee and luff vortex. For the strength of the secondary vortices the original fraction of -0.4 is retained for the lee vortex whereas the weak rebound of the luff vortex is modeled with $\Gamma_{\text {sec }} / \Gamma_{\text {prim }}=-0.2$.

\section{Adaptation of Probabilistic Envelopes}

Minor technical adjustments of classical probabilistic envelopes [23] to ground effects are described in [35]. The adjustment to selected degrees of probability is described in the following. Basis of

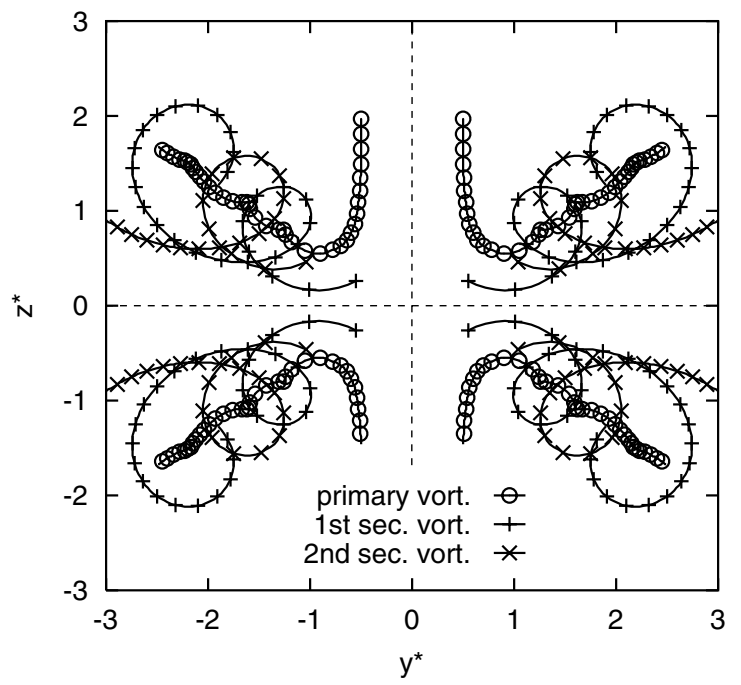

Fig. 13 Trajectories of primary and secondary vortices with image vortices for previous trajectory model. Symbols plotted every $5 \mathrm{~s}$. 


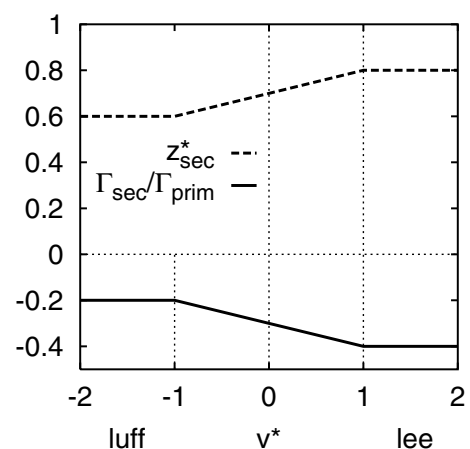

Fig. 14 Adaptation of GE trajectory model parameters to crosswind effects.

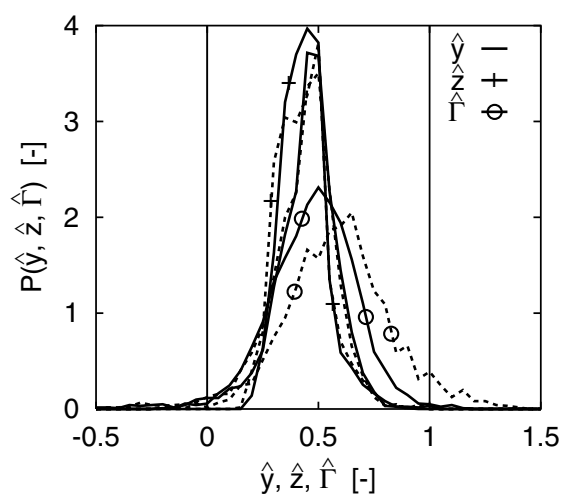

Fig. 15 Probability density distributions of measured position and circulation normalized with respect to predicted uncertainty bounds.

the model training procedure are 110 approaches to runway $25 \mathrm{R}$ accomplished at seven different days corresponding to 2449 vortex observations. The data are used to establish statistics of the relation between measured wake-vortex characteristics and the associated $\mathrm{P} 2 \mathrm{P}$ envelopes. For this purpose, the value of every measured vortex datum is normalized according to

$$
\hat{y}=\left(y_{\text {meas }}-y_{l}\right) /\left(y_{u}-y_{l}\right)
$$

Equation (5), which here is exemplarily expressed for lateral position, assigns a value of 1 to a vortex measurement situated on the predicted upper bound (index $u$ ) and the value zero to measurements on the lower bound (index $l$ ). Note that in contrast to the approach followed out of GE [25], the port and starboard vortex are normalized by their individual bounds, because in-GE systematic vortex divergence is diagnosed in lateral and vertical direction.

Figure 15 shows the probability density distributions (PDD) for lateral position, vertical position, and circulation resulting from the 110 overflights. The PDDs for lateral and vertical position of the previous model [25] applied to in GE data (dotted lines) and the new GE parameterization (solid lines) are quite similar, which indicates that the parameterization of asymmetric rebound is not essential for probabilistic prediction skill. In contrast, the respective PDDs for circulation are shifted noticeably. The frequency of nonconservative predictions $\stackrel{\text { si }}{ \pm}$ is reduced from $5.0 \%$ achieved with the out-of-GE decay model to $0.57 \%$ with the GE model.

The displacement of the circulation PDDs achieved with the outof-GE parameterization [25] and the adapted in-GE parameterization means that the former parameterization in average underestimates the observed vortex persistence. This means that for the relatively high EDR values, which are typically prevailing in ground proximity, wake vortices out of GE would decay faster compared with the actually observed decay rates in GE. As at higher altitude EDR values are usually lower [25] it cannot be concluded from this

${ }^{\ddagger}$ Nonconservative circulation predictions correspond to measurements situated above the predicted upper bound $\hat{\Gamma}=1$ and can be quantified according to $\int_{1}^{\infty} P(\hat{\Gamma}) \mathrm{d} \hat{\Gamma}$.
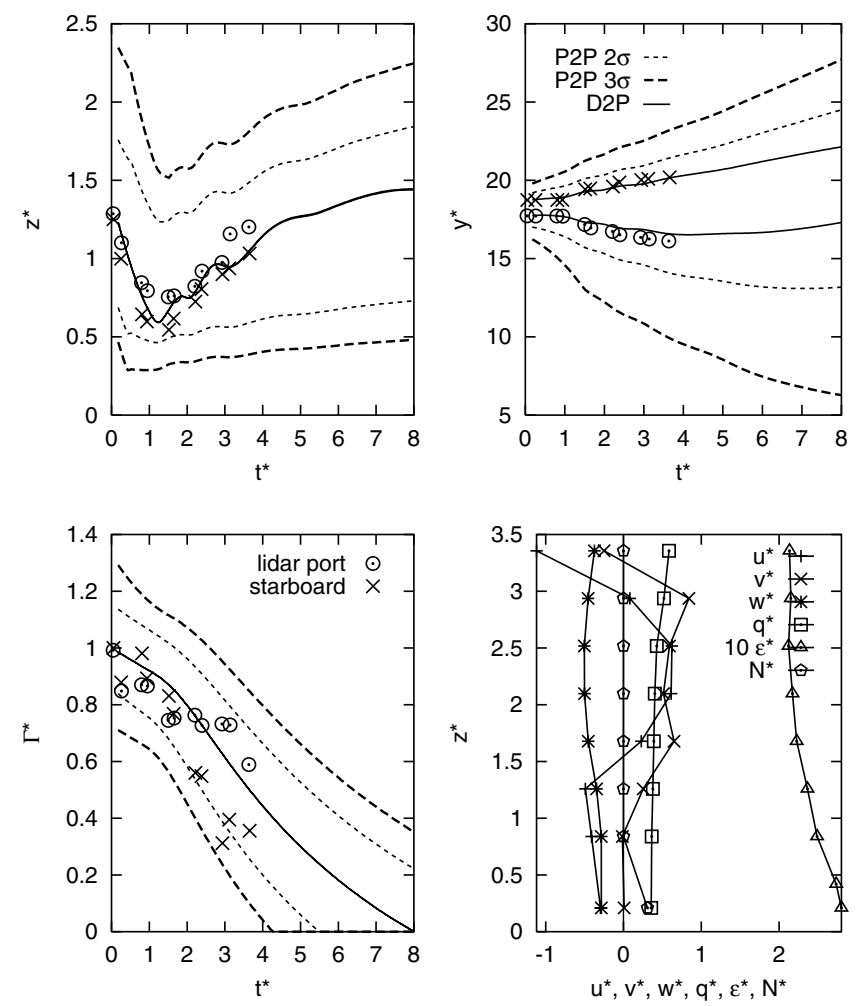

Fig. 16 Measured (symbols) and predicted (lines) wake-vortex evolution for case without crosswind.

study where decay progresses on average faster-in GE or out of GE. However, the comparison of $v_{2}^{*}$ ranges found by LES out-of-GE [23] $\left(0.0037 \leq v_{2}^{*} \leq 0.02\right)$ and in the current study in $\overline{\mathrm{GE}}$ $\left(0.002 \leq v_{2}^{*} \leq 0.004\right)$ demonstrates that at least the rapid decay phase progresses faster out of GE.

Figure 2 depicts the comparison of wake prediction and measurements for a case with a crosswind of $v^{*} \approx 1.4$. The pronounced asymmetric rebound and the lateral transport are nicely met by predictions. For comparison deterministic predictions established with the previous GE model (dashed lines) are included which feature faster decay and equal rebound of both vortices. Probabilistic envelopes for $2 \sigma$ and $3 \sigma$ are employed which correspond to probabilities of $95.4 \%$ and $99.7 \%$, respectively. The vortices remain within the $2 \sigma$ envelopes throughout their evolution which indicates that Fig. 2 displays a typical case. Figure 16 shows another case where crosswind close to the ground approximately is zero. Both prediction and observation delineate the similar rebound of port and starboard vortex.

\section{Assessment of Wake Prediction Skill}

To evaluate the performance of the suggested GE parameterizations statistics of the deviations between lidar measurements and respective deterministic predictions are compiled. The statistics is based on the 110 overflights employed in the previous section. The scoring procedure, which is described in detail in [31], was already applied to D2P predictions out of GE before [24,25]. It evaluates the root mean square deviations of measurement and prediction of the quantities $y^{*}, z^{*}$, and $\Gamma_{5-15}^{*}$ for each overflight. For an individual overflight the scoring is terminated with either the last lidar datum or when predicted circulations go to zero. From the distribution of rms values resulting from the 110 cases, the median and the 90th percentile are used to characterize the performance of the different parameterizations.

Table 1 lists scoring results for 110 approaches of heavy aircraft on 25R for the previous D2P model and different versions of GE decay parameterizations which all employ the adjusted trajectory model. Numbers in brackets left denote 91 approaches of heavy aircraft on 25L. Numbers in brackets right are based on 73 approaches of regular 
Table 1 Statistics for normalized differences between deterministic model predictions and observations

\begin{tabular}{lllcc}
\hline \hline Model & Averages & \multicolumn{1}{c}{ rms $\Delta y^{*}$} & \multicolumn{1}{c}{ rms $\Delta z^{*}$} & rms $\Delta \Gamma^{*}$ \\
\hline Previous & Median & $0.462(0.524 / 0.818)$ & $0.199(0.254 / 0.250)$ & $0.145(0.196 / 0.161)$ \\
& 90th perc. & $0.974(1.13 / 1.32)$ & $0.278(0.386 / 0.376)$ & $0.251(0.279 / 0.259)$ \\
$v_{2}^{*}=0.003$ & Median & $0.404(0.475 / 0.802)$ & $0.118(0.175 / 0.156)$ & $0.091(0.098 / 0.088)$ \\
& 90th perc. & $0.860(0.968 / 1.29)$ & $0.224(0.254 / 0.277)$ & $0.136(0.142 / 0.141)$ \\
EDR (lidar) & Median & $0.404(0.476 / 0.798)$ & $0.118(0.174 / 0.157)$ & $0.090(0.089 / 0.079)$ \\
& 90th perc. & $0.858(0.968 / 1.28)$ & $0.225(0.257 / 0.270)$ & $0.127(0.132 / 0.133)$ \\
EDR (sonic) & Median & $0.404(0.476 / 0.801)$ & $0.120(0.174 / 0.156)$ & $0.088(0.098 / 0.083)$ \\
& 90th perc. & $0.860(0.974 / 1.28)$ & $0.226(0.256 / 0.275)$ & $0.126(0.150 / 0.136)$ \\
CW & Median & $0.402(0.475 / 0.799)$ & $0.118(0.175 / 0.156)$ & $0.091(0.090 / 0.084)$ \\
& 90th perc. & $0.860(0.969 / 1.28)$ & $0.225(0.257 / 0.277)$ & $0.129(0.144 / 0.138)$ \\
\hline \hline
\end{tabular}

traffic on both runways within two morning hours. For the 110 approaches of heavy aircraft on $25 \mathrm{R}$ the table indicates that all versions of GE parameterizations (rows 2-5) improve the prediction of lateral transport, vertical transport, and circulation by about $13 \%$, $40 \%$, and $37 \%$, respectively, compared with the previous D2P model in row 1 without crosswind dependent GE trajectory model and without explicit GE decay model (comparison based on medians of approaches to 25R). The best scoring results for circulation evolution are achieved based on EDR measured by the sonic. EDR derived from lidar scores slightly worse probably because the underlying measurements were frequently contaminated by wake turbulence which degrades the EDR evaluation technique. The crosswind dependent parameterization yields almost equal scores compared with the approach based on EDR derived from lidar. The fact that reasonable scoring is also achieved with a constant effective viscosity $v_{2}^{*}=0.003$ points out that effects of turbulence and, in particular, crosswind on vortex decay, though verifiable, are weak. For the 91 approaches to runway 25L (numbers in brackets left) the ranking of circulation scoring results is modified which again illustrates that the differences between the four parameterizations are very small. Scoring results for transport are almost not influenced by different decay models.

To investigate whether the devised GE parameterization is applicable to aircraft of different sizes employing normalized vortex
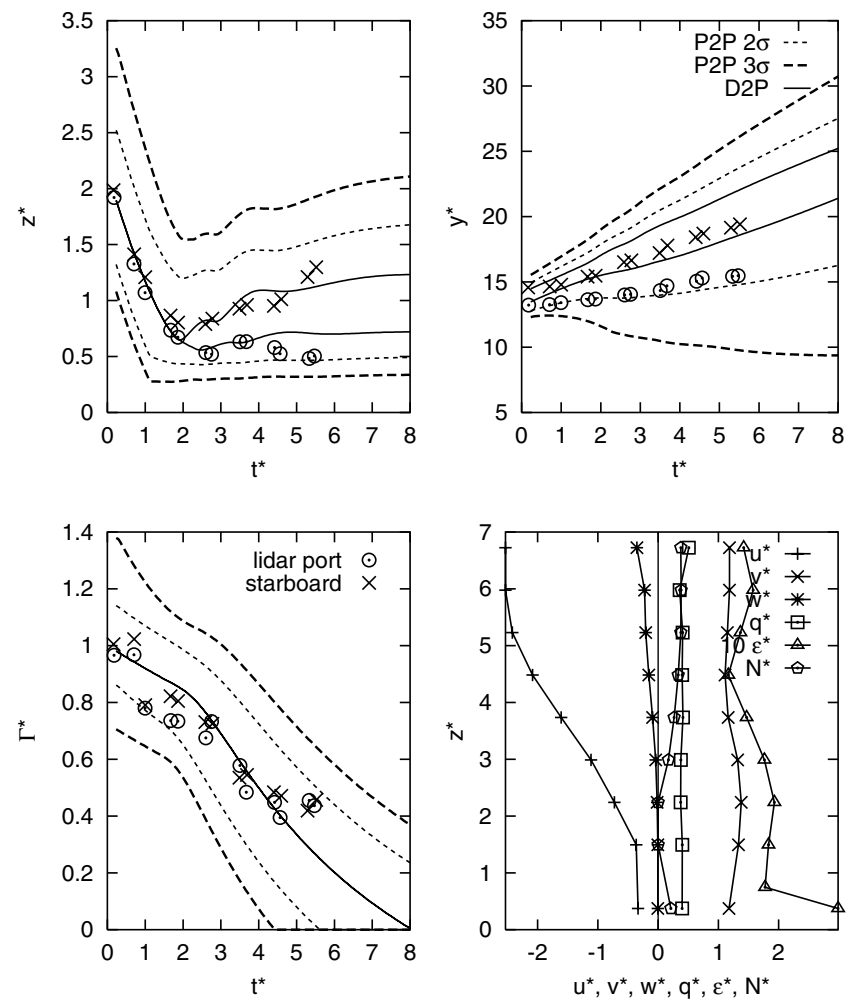

Fig. 17 Measured (symbols) and predicted (lines) wake-vortex evolution for A321 aircraft. parameters, two hours of regular traffic mix on 28 September 2004 comprising 25 different aircraft types are analyzed. The sizes of aircraft range from the Cessna Citation Excel with a wing span of $16.98 \mathrm{~m}$ to the Boeing B747-400 with a span of $64.4 \mathrm{~m}$. Ratios between maximum and minimum wing span, initial circulation, and maximum take-off weight amount to approximate factors of 3.8, 6, and 43 , respectively. The variety of considered spans have the side effect that vortices are generated within an altitude range of one to four vortex spacings above ground. This allows us to see whether the GE parameterization is sensitive to different vortex generation heights.

An inspection of the 73 cases indicates that both scaling of vortex parameters and different vortex generation altitudes do not degrade wake prediction skill (see Fig. 17). Statistics are displayed in Table 1 by numbers in brackets on the right. Although the quality of the lidar data decreases for smaller vortices, scoring results for circulation prove to be even better than for the large aircraft. This can probably be attributed to the relatively shorter times for which the evaluations of circulation from lidar data are possible for small vortices compared with vortices generated by heavy aircraft combined with the effect that typically deviations between predictions and measurements increase with vortex age. Deviations of predicted lateral positions are dominantly caused by discrepancies between measured winds and wind speeds sensed by the vortices which leads to similar absolute rms deviations for small and large aircraft. Therefore, the normalized rms deviations shown in Table 1 are substantially larger for the traffic mix compared with the heavy aircraft. Finally, scoring results for vertical position are well within the range found for heavy aircraft.

\section{Conclusions}

Though the analysis of wake-vortex behavior in ground proximity reveals a clear correlation between ambient turbulence and vortex decay, the significance of the devised turbulence parameterization turns out to be minor. Crosswind shear merely seems to introduce a slight asymmetry in the decay rate which, however, is not of practical relevance. On the other hand, the impact of crosswind shear on vortex rebound characteristics is very strong. Crosswind shear attenuates (intensifies) the formation of the luff (lee) secondary vortex which causes pronounced asymmetric rebound behavior. The impact of crosswind shear on wake-vortex evolution can be characterized by crosswind measurements at altitudes of $z=10 \mathrm{~m}$, 0.6 vortex spacings, or even higher altitudes. Crosswind strength corresponding to the initial descent speed of the vortices is sufficient to trigger the observed asymmetry.

The investigated crosswind range varies from zero to three initial vortex descent speeds $(5 \mathrm{~m} / \mathrm{s})$. For single runway applications, the considered crosswind range is sufficient because already crosswinds of two initial descent speeds prove to be effective for early clearance of a safety corridor from wake vortices. A conservative estimation of a threshold for runway clearance based on 10-min averaged crosswind magnitude measured between the runways at a standard height of $10 \mathrm{~m}$ amounts to $2.5 \mathrm{~m} / \mathrm{s}$ for a $5 \mathrm{NM}$ aircraft separation and $3.3 \mathrm{~m} / \mathrm{s}$ for a $2 \mathrm{NM}$ separation. Transport to closely spaced parallel runways was not within the scope of this study. 
Particular cases demonstrate that vortex interaction with jetlike shear layers situated above the vortices are likely to increase the rebound height. The maximum observed rebound height amounts to 2.6 initial vortex spacings. Also obstacles like parking aircraft or buildings may deflect wake vortices to higher rebound altitude. In our data the maximum height reached is two vortex spacings.

We could not clarify why in crosswind situations the interaction of the primary vortices with obviously unequally strong secondary vortices does not lead to asymmetric decay rates. Possibly, luff vortex decay is enhanced by enduring interaction with the boundary layer which may compensate for the stronger interaction between primary and secondary lee vortices at higher altitude. Here only highresolution three-dimensional simulation seems to have the potential for clarification.

Statistics of wake prediction skill indicate that decay rates adjusted by an effective viscosity which is independent from turbulence and crosswind allow for good predictions of vortex decay in ground effect. This performance can be slightly improved by applying parameterizations that depend on either crosswind or turbulence. Predictions of wake rebound and lateral transport can be significantly improved by adaptation of the strength and time of the generation of secondary vortices dependent on crosswind. Wake evolutions of a variety of differently sized aircraft types indicate that wake transport and decay can be normalized with conventional parameters.

The comparison of wake prediction skill out of ground effect (GE) in $[24,25]$ and in GE in the current study reveals that 1) decay in GE progresses distinctly slower compared with the decay rates observed during the rapid decay phase out of GE. Possibly, this difference is related to the fact that during decay in GE the wake-vortex interacts with a less strong secondary vortex $(\sim 40 \%)$ compared with the equally strong neighboring vortex out of GE. 2) Given the relatively high EDR values, which are typically prevailing in ground proximity, wake vortices out of GE would decay faster compared with the actually observed decay rates in GE. 3) Wake-vortex evolution in ground effect can be predicted with significantly increased accuracy. Comparison to [25] indicates that deterministic prediction skill is improved by $58 \%, 69 \%$, and $29 \%$ for lateral position, vertical position, and circulation, respectively. This improvement is due to the restriction of vertical transport and of ambient turbulence by a solid boundary. 4) Aloft wake-vortex prediction requires comprehensive environmental data, that is vertical profiles of wind, potential temperature, and turbulence parameters. In contrast, in ground proximity fair vortex prediction skill is achieved based on simply crosswind data.

\section{Acknowledgments}

We greatly appreciate the excellent support and work of the teams from Airbus, DFS Deutsche Flugsicherung GmbH, DLR, Fraport $\mathrm{AG}$, and METEK GmbH during and after the measurement campaign. The financial support within the DLR project Wirbelschleppe and by the European project FAR-Wake is gratefully acknowledged.

\section{References}

[1] Gerz, T., Holzäpfel, F., Bryant, W., Köpp, F., Frech, M., Tafferner, A., and Winckelmans, G., "Research Towards a Wake-Vortex Advisory System for Optimal Aircraft Spacing," Comptes Rendus Physique, Vol. 6, Nos. 4-5, 2005, pp. 501-523.

[2] De Bruin, A. C., Speijker, L. J. P., Moet, H., Krag, B., Luckner, R., and Mason, S., "S-Wake: Assessment of Wake Vortex Safety," Publishable Summary Rept., National Aerospace Laboratory NLR-TP-2003-243, Amsterdam, May 2003.

[3] Critchley, J., and Foot, P., "UK CAA Wake Vortex Database: Analysis of Incidents Reported Between 1982 and 1990," Civil Aviation Authority, CAA Paper 91, 1991.

[4] Harvey, J. K., and Perry, F. J., "Flowfield Produced by Trailing Vortices in the Vicinity of the Ground," AIAA Journal, Vol. 9, No. 8, 1971, pp. 1659-1660.

[5] Orlandi, P., "Vortex Dipol Rebound from a Wall," Physics of Fluids A, Vol. 2, No. 8, 1990, pp. 1429-1436.

[6] Schilling, V. K., "Motion and Decay of Trailing Vortices Within the
Atmospheric Surface Layer," Beiträge zur Physik der Atmosphäre, Vol. 65, No. 2, 1992, pp. 157-169.

[7] Robins, R. E., Delisi, D. P., "Potential Hazard of Aircraft Wake Vortices in Ground Effect with Crosswind," Journal of Aircraft, Vol. 30, No. 2, 1993, pp. 201-206.

[8] Luton, A., Ragab, S., and Telionis, D., "Interaction of Spanwise Vortices with a Boundary Layer," Physics of Fluids, Vol. 7, No. 11, 1995, pp. 2757-2765.

[9] Zheng, Z. C., and Ash, R. L., "Study of Aircraft Wake Vortex Behavior Near the Ground," AIAA Journal, Vol. 34, No. 3, 1996, pp. 580-589.

[10] Corjon, A., and Poinsot, T., "Behavior of Wake Vortices Near Ground," AIAA Journal, Vol. 35, No. 5, 1997, pp. 849-855.

[11] Corjon, A., and Stoessel, A., "Three-Dimensional Instability of Wake Vortices Near the Ground," AIAA Paper No. 97-1782, June-July 1997.

[12] Luton, J. A., and Ragab, S. A., "The Three-Dimensional Interaction of a Vortex Pair with a Wall," Physics of Fluids, Vol. 9, No. 10, 1997, pp. 2967-2980.

[13] Proctor, F. H., Hamilton, D. W., and Han, J., "Wake Vortex Transport and Decay in Ground Effect: Vortex Linking with the Ground," AIAA Paper No. 2000-0757, Jan. 2000.

[14] Hamilton, D. W., and Proctor, F. H., "Wake Vortex Transport in the Proximity of the Ground," Proceedings of the 19th Digital Avionics Conference, AIAA and IEEE, Philadelphia, PA, Oct. 2000, pp. 1-8.

[15] Spalart, P. R., Strelets, M. Kh., Travin, A. K., and Shur, M. L., "Modeling the Interaction of a Vortex Pair with the Ground," Fluid Dynamics, Vol. 36, No. 6, 2001, pp. 899-908.

[16] Mokry, M., "Numerical Simulation of Aircraft Trailing Vortices Interacting with Ambient Shear or Ground," Journal of Aircraft, Vol. 38, No. 4, 2001, pp. 636-643.

[17] Liu, H.-T., Hwang, P. A., and Srnsky, R. A., "Physical Modeling of Ground Effects on Vortex Wakes," Journal of Aircraft, Vol. 29, No. 6, 1992, pp. 1027-1034.

[18] Köpp, F., "Doppler Lidar Investigation of Wake Vortex Transport Between Closely Spaced Parallel Runways," AIAA Journal, Vol. 32, No. 4, 1994, pp. 805-810.

[19] Hallock, J. N., Osgood, S. P., and Konopka, J., "Wake Vortex Effects on Parallel Runway Operations," AIAA Paper No. 2003-0379, 2003.

[20] Doligalski, T. L., Smith, C. R., and Walker, J. D. A., "Vortex Interactions with Walls," Annual Review of Fluid Mechanics, Vol. 26, Jan. 1994, pp. 573-616.

[21] Holzäpfel, F., Hofbauer, T., Darracq, D., Moet, H., Garnier, F., and Ferreira Gago, C., "Analysis of Wake Vortex Decay Mechanisms in the Atmosphere," Aerospace Science and Technology, Vol. 7, No. 4, 2003, pp. 263-275.

[22] Burnham, D. C., and Hallock, J. N., "Measurements of Wake Vortices Interacting with the Ground," AIAA Paper No. 98-0593, Jan. 1998.

[23] Holzäpfel, F., "Probabilistic Two-Phase Wake Vortex Decay and Transport Model," Journal of Aircraft, Vol. 40, No. 2, 2003, pp. 323 331.

[24] Holzäpfel, F., and Robins, R. E., "Probabilistic Two-Phase Aircraft Wake-Vortex Model: Application and Assessment," Journal of Aircraft, Vol. 41, No. 5, 2004, pp. 1117-1126.

[25] Holzäpfel, F., "Probabilistic Two-Phase Aircraft Wake-Vortex Model: Further Development and Assessment," Journal of Aircraft, Vol. 43, No. 3, 2006, pp. 700-708.

[26] Frech, M., and Holzäpfel, F., "Skill of an Aircraft Wake-Vortex Transport and Decay Model Using Short-Term Weather Prediction and Observation," Proceedings of the 12th Conference on Aviation, Range, and Aerospace Meteorology, Paper 6.9, American Meteorological Society, Boston, Jan. 2006.

[27] Köpp, F., Rahm, S., and Smalikho, I., "Characterization of Aircraft Wake Vortices by 2-m $\mu$ Pulsed Doppler Lidar," Journal of Atmospheric and Oceanic Technology, Vol. 21, No. 2, 2004, pp. 194-206.

[28] Smalikho, I., Köpp, F., and Rahm, S., "Measurement of Atmospheric Turbulence by 2- $\mu \mathrm{m}$ Doppler Lidar," Journal of Atmospheric and Oceanic Technology, Vol. 22, No. 11, 2005, pp. 1733-1747.

[29] Lamb, Sir H., Hydrodynamics, Dover Publications, New York, 1945, p. 590.

[30] Holzäpfel, F., Gerz, T., Köpp, F., Stumpf, E., Harris, M., Young, R. I., and Dolfi-Bouteyre, A., "Strategies for Circulation Evaluation of Aircraft Wake Vortices Measured by Lidar," Journal of Atmospheric and Oceanic Technology, Vol. 20, No. 8, 2003, pp. 1183-1195.

[31] Robins, R. E., and Delisi, D. P., "Wake Vortex Algorithm Scoring Results," NASA CR-2002-211745, June 2002.

[32] Holzäpfel, F., Hofbauer, T., Gerz, T., and Schumann, U., "Aircraft Wake Vortex Evolution and Decay in Idealized and Real Environments: Methodologies, Benefits and Limitations," Advances in LES of Complex Flows, edited by R. Friedrich and W. Rodi, Fluid 
Mechanics and Its Applications, Vol. 65, Kluwer Academic Publishers, Dordrecht, The Netherlands, 2002, pp. 293-309.

[33] Gurke, T., and Lafferton, H., "The Development of the Wake Vortices Warning System for Frankfurt Airport: Theory and Implementation," Air Traffic Control Quarterly, Vol. 5, No. 1, 1997, pp. 3-29.

[34] Robins, R. E., Delisi, D. P., and Greene, G. C., "Algorithm for Prediction of Trailing Vortex Evolution," Journal of Aircraft, Vol. 38,
No. 5, 2001, pp. 911-917.

[35] Holzäpfel, F., and Steen, M., "Aircraft Wake-Vortex Evolution in Ground Proximity: Analysis and Parameterization," AIAA Paper 20061077, Jan. 2006.

A. Plotkin Associate Editor 